- General medical council (GMC) trainees' survey highlighted red flags in training: workload, access to teaching and senior supervision.

- Discrepancy in ability to work within contracted hours between inpatient teams.

- Low morale amongst junior doctor (JD) workforce.

- Deanery training posts allocations not keeping pace with the change in medical inpatient demographics.

- This project aimed to:

- Benchmark workforce and workload against standards from the Royal College of Physicians (RCP).

- Identify root causes for variation.

- Suggest actions to future proof the medical workforce.

Methods

- Two-week audit of activity of 20 medical teams across the trust applying the RCP tool to estimate workload against recommended workforce.

- Total JD ward work-hours per week within each tier of seniority calculated.

- Variation of workload to workforce ratio determined.

- Questionnaires sent to all JDs regarding their perceptions of challenges.

Results

- Wide variation of workload to workforce ratio.

- Tier 1 staffing is above minimum RCP recommendations in all teams.

- Tier 2 presence on medical wards is on average $1 / 4$ RCP recommendation.

- The reasons for non-compliance with contracted hours and dissatisfaction with training cannot be explained by shortage of tier 1 doctors.

Conclusions The finding of adequate overall levels of staffing and outdated allocation is important. Addressing historical workload imbalances will impact significantly upon safety, quality of care, training and morale. JD staffing is often cited as inadequate. While this may be true, it is also likely that JD workload is similarly inefficient across the country. We hope this work will encourage others to identify and improve inefficiencies in JD workload.

\section{9 'WHO CARES FOR THE CARERS?' IMPROVING JUNIOR DOCTOR WELLBEING AND MORALE AT ROYAL SURREY COUNTY HOSPITAL (RSCH), GUILDFORD}

Emma Hadley, Mary Stevenage, Jane Tilley. Royal Surrey County Hospital, Guildford

10.1136/leader-2019-FMLM.29

Four in five junior doctors (JDs) regularly experiencing excessive work-related stress and one in four state their job has a serious negative impact on their mental health.1 Addressing these issues improves the quality of service delivered to patients, patient safety and outcomes.2 Its importance is reflected in the inclusion of staff wellbeing as a top priority for Royal Surrey County Hospitals (RSCH) five-year strategic goals. A trust-wide project; spearheaded by a Leadership Fellow and supported by the Director of Medical Education and BMA, was conducted to assess the magnitude of this problem locally and identify simple, sustainable and affordable Methods to address these issues.
Initially a JD Wellbeing Questionnaire was distributed, JD Wellbeing Forum and 1:1 discussions for anecdotal information conducted. Review of recent national guidelines and recommendations related to NHS staff wellbeing were examined, and models of wellbeing-support at other organisations were appraised. The Results from these were collated and led to the identification of four main areas that required improvement along with strategies to address these.

The success of the project relied on early identification, networking, and involvement of key stakeholders, ranging from JDs to senior members of staff, clinical and non-clinical such as the Medical Director and Director of Human Resources. Their involvement helped maintain momentum, enable Results of greater magnitude and ensure sustainability. Widespread publicity of the project gained buy-in from staff and although the project predominantly focussed on JDs, the project resonated with other staff groups, resulting in an allencompassing project, trust-wide change and a global shift in culture.

This project brands $\mathrm{RSCH}$ as a pioneer in this revolutionary transformation of attitude towards staff wellbeing. The concepts and strategies outlined in this project, based on national recommendations, should be replicated at other trusts.

\section{DO JUNIOR-DOCTOR LED FOCUS GROUPS IMPROVE UNDERSTANDING OF THE ROLE OF CQC IN REGULATION OF HEALTHCARE?}

Helen Grote, ona Thorne. Care Quality Commission, 151 Buckingham Palace Road. London. UK

10.1136/leader-2019-FMLM.30

Introduction Junior doctors have been described as the 'eyes and ears' of hospitals, ${ }^{1}$ but most feel disengaged from CQC inspections. $^{2}$ During 2018-2019 CQC piloted a series of junior doctor focus groups, led by junior doctor specialist advisors (SpAs).

The aim of these focus groups was to i) Improve the intelligence received from junior doctors ii) Increase engagement of junior doctors with the inspection process and iii) Increase understanding about the role of CQC.

Method Training materials were provided to junior doctor SpAs in the form of a video about the CQC, and a guide to running focus groups. Sixteen NHS trusts were visited (7 mental health, 9 acute).

A 10 minute presentation about CQC was given immediately prior to the focus group. Evaluation was undertaken using an online survey.

Results Thirty junior doctors completed the online survey. All were aware that CQC inspects hospitals, but only 19 (63\%) were aware that CQC also regulates social care and dentists. Most (26/30) had never read the inspection report for their trust. All rated regulation as 'important' or 'very important', but over half felt disengaged from the inspection process. Most (26/30) agreed that the presentation improved their understanding of the CQC, and provided the CQC with accurate information about the trust (28/30).

Conclusion Inspectors and junior doctors found focus groups useful for informing the inspection process, and for improving understanding of the CQC. CQC is continuing this 
programme of focus groups to increase engagement with junior doctors and inform the rating of providers at well-led. In addition, this work will provide junior doctors with more opportunities for leadership and engagement with trust boards. ${ }^{3}$

\section{REFERENCES}

1. https://www.nhs.uk/nhsengland/bruce-keogh-review/documents/outcomes/keoghreview-final-report.pdf

2. Data collected by CQC, 2018-2019.

3. https://improvement.nhs.uk/documents/1884/NHS-8-high-impacts-A4v5Bm_with_stickynotes_5_7dglFbL.pdf

\section{Developing Effective Leaders}

\section{PERIOPERATIVE MEDICINE FOR OLDER PEOPLE UNDERGOING SURGERY (POPS) FELLOWSHIP: DEVELOPING LEADERS IN GERIATRIC PERIOPERATIVE MEDICINE}

${ }^{1}$ Rajni Lal*, ${ }^{2}$ Emily Jasper, ${ }^{1}$ Cathryn Mainwaring, ${ }^{3}$ Judith Partridge, ${ }^{3}$ Jugdeep Dhesi. 'Specialist Registrar, Ageing and Health, Guy's and St Thomas' NHS Foundation Trust, London UK: ${ }^{2}$ Research Registrar, Ageing and Health, Guy's and St Thomas' NHS Foundation Trust, London UK; ${ }^{3}$ Geriatrician, Ageing and Health, Guy's and St Thomas' NHS Foundation Trust, London UK

\subsection{6/leader-2019-FMLM.31}

Background Perioperative medicine (POM) for older people is an emerging geriatric subspecialty where effective leadership is essential to support national growth. POPS is an evidenced-based, geriatrician-led, multidisciplinary team, providing comprehensive geriatric assessment(CGA) for older patients undergoing elective and emergency surgery. There is heterogeneity observed in the UK, with only $53 \%$ of trusts providing POPS services in 2018. ${ }^{1}$ UK surveys demonstrate current provision does not meet demand, meaning developing leaders in POM is vital for scaling POPS services.

Method The GSTT POPS fellowship was developed in 2012 to provide Specialist Registrars the opportunity to spend a year under the supervision of POM geriatricians. Fellows develop experience in CGA before elective surgery and inpatient care for all older surgical patients. The curriculum outlines expected knowledge, behaviours and opportunities available. Leadership skills are developed through independent work, junior supervision, research and mentorship from geriatricians.

Results 10 trainees have completed the fellowship, with 6 now working POPS Consultants. Achievements include; 16 quality improvement projects, 5 publications, 5 awarded grants, a Darzi fellowship and representation on national bodies (eg. NELA). The wider impact is seen through UK POPS expansion and improved outcomes for older patients in NELA. ${ }^{1,2}$

Conclusion In the context of workforce shortages, difficulties in geriatrician recruitment requires service flexibility. This led to the development of a POPS fellowship, now producing future POM leaders.

\section{REFERENCES}

1. Joughin A, Partridge J, O'Halloran T, et al. Where are we now in perioperative medicine? Results from a repeated UK survey of geriatric medicine delivered services for older people. Age Ageing 2019;48:458-462.

2. Fourth Patient Report - National Emergency Laparotomy Audit(NELA). Available from:http://www.nela.org.uk/Fourth-Patient-Audit-Report[Accessed;2019,May 24]

\section{Leading Innovation and Improvement}

\section{TEA BREAK TALK - A WELLBEING INITIATIVE FOR JUNIOR DOCTORS}

Bernadetta Btari Adityani, Cristina Musat. University Hospitals Plymouth, NHS Trust, UK

\subsection{6/leader-2019-FMLM.32}

Background The 2019 BMA report on 'Caring for the mental health of the medical workforce' found that $80 \%$ of doctors are at high risk of burnout with junior doctors being most at risk. The 2018 GMC Training Environment Report also found that $25 \%$ of doctors in training felt burnout associated with high workloads, rota gaps, and a lack of a supportive environment.

Method Members of the Junior Doctors Representative Committee at University Hospitals Plymouth NHS Trust, launched a bi-monthly initiative called Tea Break Talk, with BMA funding. Through open group discussions and questionnaires we assessed perceptions around seeking support from Clinical/Educational Supervisors and we supported the wellbeing of junior doctors locally by signposting available services and peer-led discussion/reflection.

Results Over 3 months, we obtained feedback from doctors ranging from F1-ST6 levels. All responders found these sessions to be beneficial and a safe platform to discuss concerns regarding their wellbeing and professional development. 44\% of the attendees were not aware of the existing counselling services; only $48 \%$ felt comfortable discussing stressful scenarios with their supervisors; one third of responders would discuss other personal or health-related concerns with their supervisors and almost $20 \%$ felt that doing so would reflect badly on them.

\section{Developing Effective Leaders}

\section{CLINICAL SENATE FELLOWSHIPS AS A FORUM FOR DEVELOPING FUTURE CLINICAL AND MEDICAL LEADERS}

${ }^{1}$ Rebecca Hall, ${ }^{2}$ Christopher J Miller, ${ }^{3}$ Remi Popoola, ${ }^{4}$ Lucy Gavens, ${ }^{3}$ Elizabeth Gonzalez Malaga, ${ }^{2}$ Eyad Issa, ${ }^{5}$ Emma Orrock, ${ }^{5}$ Alyson Evans, ${ }^{5}$ Lara Harrison, ${ }^{2,5}$ Ashley Dennison, ${ }^{6}$ Aly Rashid. ${ }^{1}$ Roseberry Medical Centre Loughborough, UK; ${ }^{2}$ University Hospitals of Leicester NHS Trust, UK; ${ }^{3}$ Northamptonshire Healthcare NHS Foundation Trust, UK; ${ }^{4}$ Derbyshire County Council, UK; ${ }^{5}$ East Midlands Clinical Senate, UK; ${ }^{6}$ NHS England and NHS Improvement - Midlands, UK

\subsection{6/leader-2019-FMLM.33}

Aims The East Midlands Clinical Senate is one of twelve regional clinical senates. Clinical senates act as a forum of multi-professional clinical leaders who can offer independent and objective clinical advice to support commissioners and other stakeholders to make the best decisions about health care for their local populations. The East Midlands Clinical Senate recognised this would provide a unique opportunity to recruit clinical research fellows, creating the opportunity to support leadership development and enhance our ability to produce high quality research to support evidence-based decision making among health and social care leaders.

Methods A competitive recruitment process engaged six clinical fellows from a variety of specialties, for one session a week over a 12 month period. The clinical fellows were 